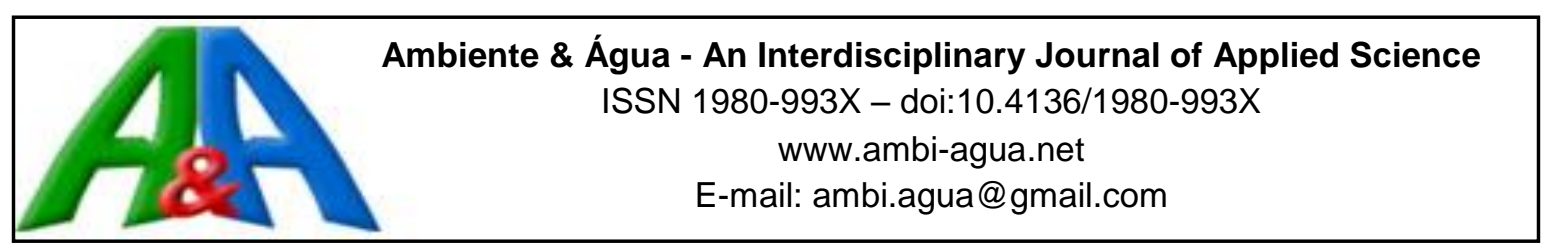

\title{
Riscos socioeconômicos e ambientais em municípios banhados pelos afluentes do Rio Amazonas
}

\author{
doi:10.4136/ambi-agua.2013
}

Received: 11 Oct. 2016; Accepted: 26 Jun. 2017

\author{
Eliane de Castro Coutinho ${ }^{1,2 *}$; Edson José Paulino da Rocha ${ }^{2}$; \\ Aline Maria Meiguins Lima² ${ }^{2}$ Hebe Morganne Campos Ribeiro'; \\ Lucy Anne Cardoso Lobão Gutierrez ${ }^{1}$; Ana Júlia Soares Barbosa ${ }^{1}$; \\ Gleicy Karen Abdon Alves Paes ${ }^{1}$; Carlos José Capela Bispo ${ }^{1}$; \\ Paulo Amador Tavares ${ }^{1}$ \\ ${ }^{1}$ Universidade do Estado do Pará (UEPA), Belém, PA, Brasil \\ Departamento de Engenharia Ambiental do Centro de Ciências Naturais e Tecnologia (UEPA) \\ ${ }^{2}$ Universidade Federal do Pará (UFPA), Belém, Pará, Brasil \\ Instituto de Geociências (UFPA) \\ *Autor correspondente: e-mail: elianerik@ gmail.com, \\ eprocha@ufpa.br, alinemeiguins@gmail.com, hebemcr@gmail.com, \\ lucyannegutierrez@gmail.com, ajsbarbosa@gmail.com, \\ gleicykaren@yahoo.com.br, cjcapela@yahoo.com.br, \\ ptavares_20@hotmail.com
}

\section{RESUMO}

Os municípios da Amazônia brasileira são constantemente influenciados por secas e cheias, onde riscos socioeconômicos e ambientais afetam principalmente a população ribeirinha. Esses extremos de precipitação causam mudanças severas na hidrologia dos rios tanto em escala temporal quanto espacial. Sendo assim, o objetivo deste estudo foi determinar o índice do risco socioeconômico e ambiental dos municípios que recebem influência dos afluentes e da calha principal do Rio Amazonas, comparando com eventos extremos de precipitação. Utilizaram-se dados mensais e anuais de precipitação de 1982-2012 e dados sociais (população urbana, idosa, feminina e de crianças, nível de rendimento e de escolaridade) de 2010 para 47 municípios na Bacia Amazônica. Contudo, conclui-se que o risco foi maior durante eventos de cheia, principalmente, nos menores estados (Acre e Roraima) e maior vulnerabilidade nos maiores estados (Amazonas e Pará). Assim, as populações nos municípios ao longo do Rio Amazonas possuem risco socioeconômico e ambiental de moderado a muito forte devido à vulnerabilidade alta associada com a urbanização e com a pobreza, além de ameaça de enchentes e estiagens variando de moderada a alta.

Palavras-chave: eventos extremos, região amazônica, vulnerabilidade.

\section{Socioeconomic and environmental risks in municipalities bathed by tributaries of the Amazon River}

\begin{abstract}
Municipalities in the Amazon are constantly affected by droughts and floods, and these socioeconomic and environmental risks mainly affect the local population. These precipitation extremes cause severe changes in rivers' hydrology, on both a temporal and a spatial scale. The
\end{abstract}


intended objective of this study therefore was to determine the socioeconomic and environmental risk of municipalities affected by the tributaries and by the main channel of the Amazon River in relation to extreme precipitation events. We used monthly and annual precipitation data from 1982 to 2012 and social data from 2010 (urban, elderly, female and child populations, income and education level) for 47 localities in the Amazon Basin. We concluded that the risk was highest during flood events, particularly in smaller states (Acre and Roraima), and that vulnerability was greater in larger states (Amazonas and Pará). However, the population in the municipalities along the Amazon River have moderate to very strong socioeconomic and environmental risk because of the vulnerability associated with high urbanization and poverty, and threat of floods and droughts ranging from moderate to high.

Keywords: amazon region, extreme events, vulnerability.

\section{INTRODUÇÃO}

Hoje os estados brasileiros possuem uma taxa de ocupação urbana desordenada muito alta de, em média, $80 \%$. Porém se fossem aplicadas as legislações urbanas e os planos diretores dos municípios, essa realidade seria diferente, pois com o planejamento urbano poder-se-ia propor ações adequadas para construir cidades mais sustentáveis, com justiça e qualidade de vida para todos. Além disso, Assad (2011) confirmou que $75 \%$ da população mundial entre os anos de 1980 e 2000 sofreram com a ocorrência de fenômenos, como: ciclones, furacões e enchentes, corroborando com os estudos de Braga et al. (2006), pois afirmaram que diversos tipos de desastres naturais em cidades que afetam populações inteiras provocam danos intangíveis e econômicos.

Desastres naturais, como as enchentes e inundações, hoje são mais percebidas devido a falta de planejamento e infraestrutura urbana. Leal e Souza (2011) desenvolveram uma pesquisa com o objetivo de mapear os desastres naturais associados às enchentes e inundações registradas na Amazônica, e comprovaram que tais problemáticas tornam diversas áreas vulneráveis, aumentando os riscos de danos e prejuízos à população, podendo causar mortes com o desmoronamento de terras e a proliferação de doenças hídricas. Esses autores ainda mostraram que $73 \%$ de todo o estado do Pará teve seus municípios atingidos por desastres, sendo as inundações as mais frequentes, pois $95 \%$ desses desastres naturais estão relacionados com as variações dos índices pluviométricos.

Os desastres naturais não são sentidos igualmente por todos, pois as mulheres, as crianças e os idosos são os mais afetados, além das pessoas mais pobres. Esses parâmetros são utilizados para determinar o risco de vulnerabilidade de uma região, que é segundo Brauch (2005), a suscetibilidade ao perigo ou dano, ou também, conforme O’Riordan e Stoll-Kleemann (2002), é a incapacidade de uma sociedade em evitar o perigo relacionado às catástrofes naturais. Além dos desastres naturais, o que também afeta esse grupo da população são as áreas de degradação ambiental que normalmente são as áreas mais pobres e com privação social, pois crescem desordenadamente sem infraestrutura básica. Assim, Alves (2006), em seu estudo para a metrópole paulistana, afirma que a vulnerabilidade ambiental é um fator relevante na distribuição espacial das situações de pobreza e privação social, pois em seus resultados a alta vulnerabilidade social estava relacionada com menor rendimento em locais degradados.

Gonçalves et al. (2014) também comprovam, em seu estudo, que os grupos mais pobres são os mais vulneráveis, devido as condições ambientais e sociais mais precárias, além da falta de apoio do Estado ou de outras fontes externas. Apesar da região Amazônica ser rica em recursos naturais e possuir uma grande biodiversidade, seu Índice de Desenvolvimento Humano (IDH) é o mais baixo do país, pois segundo Pinho et al. (2015), 42\% da população na Amazônia é afetada pela pobreza, enquanto a taxa de pobreza de todo o Brasil é de $28,8 \%$. Além disso, o analfabetismo afeta $13 \%$ da população amazônica, contra $11 \%$ do total do país, e também deve 
ser considerado o fator da urbanização, que hoje é de 80\% (Assad, 2011; Parry et al., 2010; Araujo e Léna, 2010).

Nos últimos 30 anos ocorreram cinco eventos de seca (estiagem): em 1983, estudado por Marengo et al. (2008), em 1998 por Marengo et al. (2008) e Espinoza et al. (2011), em 2005 e 2010 por Espinoza et al. (2011); e quatro eventos de cheias (enchentes): em 1989, 1999 e 2009 estudadas por Marengo et al. (2012) e em 2012 estudada por Satyamurty et al. (2013) e Marengo et al. (2013).

A seca de 2005, segundo Marengo et al. (2008), foi a maior ocorrida na Amazônia ocidental nos últimos cem anos, afetando a população ao longo do Rio Amazonas e seus afluentes, oeste e sudoeste, o Solimões e o rio Madeira, deixando o maior rio do mundo quase sem água, porém não afetou o centro e leste da Amazônia. Rolim et al. (2006) também estudaram os impactos gerados pela ação de fenômenos naturais na região Amazônica devido à urbanização acelerada, comprovando que o monitoramento desses impactos, em áreas de risco e vulneráveis, é objeto de vários estudos científicos, pois preparar a população para enfrentar tais impactos tornou-se um grande desafio da sociedade e do Estado brasileiro.

Além das secas, a Amazônia também foi afetada por enchentes, como a de 2009 que teve a magnitude e duração maior nas últimas décadas; porém não maior que a de 2012, com chuvas torrenciais no norte e leste da Amazônia durante o verão austral de 2008-2009, provocando cheias no rio principal e em seus afluentes. O Rio Negro chegou a uma cota nunca vista nos últimos 107 anos, e as chuvas no norte e noroeste da Amazônia começaram prematuramente com um período mais longo que o normal. Segundo Marengo et al. (2012) esse episódio foi devido a migração anômala da Zona de Convergência Intertropical (ZCIT) durante maio e junho de 2009, pelo aquecimento anômalo das águas da superfície do Atlântico Sul; e as enchentes registradas em 1989 e 1999 ocorreram mais cedo do que as inundações normais dos afluentes do norte da Amazônia, fazendo com que a vazão coincidisse com os picos de inundações dos afluentes do sul.

Filizola et al. (2014) também estudaram a enchente de 2009, e afirmaram que esse evento mostrou uma nova forma de lidar com os riscos e desastres na bacia amazônica, pois mais de 238 mil moradores de 38 municípios foram afetados causando um efeito drástico na população local.

É importante suscitar que nas últimas décadas as preocupações com as injustiças ambientais aumentaram principalmente quando se refere à distribuição dos riscos ambientais frente às populações de baixa renda, conforme Cartier et al. (2009). Contudo o conceito de injustiça ambiental, por Acselrad et al. (2004) é:

[...] ]o mecanismo pelo qual sociedades desiguais, do ponto de vista econômico e social, destinam a maior carga dos danos ambientais do desenvolvimento às populações de baixa renda, aos grupos sociais discriminados, aos povos étnicos tradicionais, aos bairros operários, às populações marginalizadas e vulneráveis.

Assim a vulnerabilidade, segundo Pinho et al. (2015) é um processo dinâmico devido às desigualdades no acesso e distribuição dos recursos, causando a marginalização social em diferentes escalas. Assim, a governança adequada teria essa marginalização potencial e deveria agir para reduzí-la, melhorando os meios de subsistência regional e a educação, diminuindo a pobreza, diminuindo o êxodo rural e com isso diminuindo a urbanização acelerada e desordenada, a qual é um dos grandes responsáveis pelos riscos ambientais, que aumentam ainda mais a vulnerabilidade da população. Já segundo Alves (2006) e Sena et al. (2012), vulnerabilidade socioambiental é uma parte da população fortemente carente e necessitada (vulnerabilidade social), juntamente com áreas sujeitas a risco ou degradação ambiental 
(vulnerabilidade ambiental). Assim pode-se afirmar que existe uma relação direta entre riscos ambientais e precariedade no acesso aos serviços públicos (falta de infra-estrutura urbana - água, saneamento, coleta de lixo, canalização de cursos de água, entre outros).

Sena et al. (2012), em seu estudo, afirmam que as zonas mais vulneráveis utilizam a renda, densidade populacional e educação como indicador, além disso os resultados desenvolvidos nos estudos desses autores na bacia do rio Acre revelaram que os eventos extremos, principalmente as inundações que ocorrem nas regiões mais densamente urbanizadas, agravam a vulnerabilidade social, principalmente nas áreas mais pobres.

Risco é diferente de vulnerabilidade, como Esteves (2011) explica em seu artigo, pois está relacionado aos acidentes naturais, segurança pessoal, saúde, condições de habitação, trabalho, transporte, condição da criança e do adolescente, entre outros, ou seja, pode ser encarado como uma situação de perigo.

Apesar da Amazônia abrigar uma pequena parte da população mundial (cerca de 5\%), sua relevância global está na sua riqueza em termos de biodiversidade, influência na umidade e nas chuvas de todo o continente Sul Americano. Além disso, a Amazônia chama a atenção devido às mudanças climáticas, pois sua hidrologia e sua população são fortemente afetadas por eventos extremos, principalmente os associados à precipitação, como constatado por Guimberteau et al. (2013).

Assim o objetivo desse estudo é determinar o risco socioeconômico e ambiental dos municípios que recebem influência dos afluentes e da calha principal do Rio Amazonas, comparando os eventos de seca e de cheia aos dados socioeconômicos que mais mostram a vulnerabilidade da população às catástrofes ambientais. Sendo assim, foi necessária a determinação do índice de vulnerabilidade socioeconômico e a ameaça de cheia e seca.

\section{MATERIAL E MÉTODOS}

A Região Amazônica possui uma considerável densidade de redes fluviométricas, porém muitas séries de dados possuem falhas e não operam no mesmo período de tempo. Assim optou-se por utilizar 47 postos fluviométricos com homogeneidade de dados durante o período de 1982 e 2012, totalizando 31 anos.

$\mathrm{Na}$ análise da vulnerabilidade, das ameaças e dos riscos socioeconômicos e ambientais nos municípios que recebem influência dos afluentes do Rio Amazonas, bem como da calha principal, foram utilizados dados censitários do IBGE (2010) e dados de vazão mensais da ANA (2013).

Entre os dados censitários, representando a parte socioeconômica da pesquisa, utilizou-se o total de habitantes de cada município (PT), o total de habitantes da área urbana (PU), o total da população feminina (PF), o total da população de crianças até 4 anos de idade (PC), o total da população de idosos com mais de 60 anos de idade (PI), o total da população com rendimento até 2 salários mínimos (PR) e total da população com o ensino fundamental incompleto (PE) do ano de 2010. Os dados fluviométricos, que representam a análise ambiental do estudo, foram tratados mensal e anualmente.

A metodologia desta pesquisa está dividida em três etapas: a vulnerabilidade socioeconômica, a ameaça ambiental e o risco socioeconômico e ambiental.

\subsection{Vulnerabilidade socioeconômica}

Para a construção do Índice de Vulnerabilidade Socioeconômica e Ambiental (IVS) foi necessário determinar as variáveis que mais representam as vulnerabilidades que uma 
população sofre nas localidades onde residem, conforme as características e a importância da utilização de cada uma delas, levando em consideração a metodologia de Szlafstein et al., 2010.

a) PU: Proporção da população total urbana do Município sobre PT. Katayama (1993) sugere que se uma região é inabitada, a vulnerabilidade social pode ser nula;

b) PC: Proporção da população total de crianças com menos de 4 anos de idade sobre PT. Este grupo populacional está usualmente entre as primeiras vítimas durante os desastres, devido a alta dependência de seus familiares. Os efeitos dos desastres abarcam desde os físicos até psicológicos, causados pelo pânico, e as incertezas associadas à evacuação (Fleck e Wagner, 2003);

c) PI: Proporção da população total de idosos com mais de 60 anos sobre PT. A Organização Mundial da Saúde classifica cronologicamente como idosas, as pessoas com mais de 60 anos de idade em países em desenvolvimento. As pessoas idosas sofrem quando suas casas devem ser evacuadas e elas encontram dificuldade para recuperar-se da perda dos seus pertences e outras economias, além da dificuldade de locomoção com rapidez, conforme Quarantelli e Popov (1994);

d) PF: Proporção da população total feminina sobre PT. As mulheres representam em muitos casos o sustento emocional, biológico, e econômico das famílias (Fleck e Wagner, 2003), além de quase sempre estarem acompanhadas de crianças que são o grupo populacional mais vulnerável;

e) PE: Proporção da população total de pessoas com o ensino fundamental incompleto sobre PT. Pessoas que ainda não cumpriram a primeira etapa do ensino fundamental no Brasil têm dificuldades de entenderem a importância de evacuação dos locais com vulnerabilidade alta, preocupando-se mais com os bens materiais do que os bens intangíveis; e

f) PR: Proporção da população total com rendimento de até 2 salários mínimos sobre PT. A pobreza está relacionada à marginalização social e a carência de acesso a recursos e às adaptações, transformando este grupo populacional no alvo principal dos impactos dos desastres e das mudanças climáticas (Szlafsztein et al., 2010).

Após determinação das variáveis e identificação da importância de cada uma delas, a classificação de vulnerabilidade das mesmas foi agrupada na Tabela 1, conforme Szlafstein et al. (2010).

Tabela 1. Classificação da vulnerabilidade socioeconômica.

\begin{tabular}{lccccccc}
\hline Vulnerabilidade & Classificação & $\begin{array}{c}\text { População } \\
\text { Urbana (PU) }\end{array}$ & $\begin{array}{c}\text { População de } \\
\text { Crianças (PC) }\end{array}$ & $\begin{array}{c}\text { População de } \\
\text { Idosos (PI) }\end{array}$ & $\begin{array}{c}\text { População } \\
\text { Feminina (PF) }\end{array}$ & $\begin{array}{c}\text { Nível de } \\
\text { Escolaridade (PE) }\end{array}$ & $\begin{array}{c}\text { Nível de } \\
\text { Rendimento (PR) }\end{array}$ \\
\hline Baixa & 0 & Até 5\% & Até 5\% & Até 5\% & Até 10\% & Até 30\% & Até 10\% \\
Moderada & 0,5 & 5 a 10\% & 5 a 10\% & 5 a 10\% & 10 a 20\% & 30 a 50\% & 10 a 20\% \\
Alta & 1 & Mais 10\% & Mais de 10\% & Mais de 10\% & Mais de 20\% & Mais de 50\% & Mais de 20\% \\
\hline
\end{tabular}

Fonte: Szlafstein et al. (2010).

Após a identificação da vulnerabilidade socioeconômica para cada aspecto, foi utilizada a Equação 1 para determinar o IVS.

$$
I V S=\frac{(P U+P C+P I+P F+P E+P R)}{6}
$$




\subsection{Ameaça ambiental}

A segunda etapa foi feita para a determinação da ameaça ambiental entre 1982 e 2012, levando em consideração os períodos de seca e cheia. Os dados de vazão $\left(\mathrm{m}^{3} \mathrm{~s}^{-1}\right)$ foram normalizados aplicando a Equação 2, variando de -1 a 1, onde os valores negativos referem-se aos eventos de seca, os valores positivos aos eventos de cheia e o zero refere-se a localidades sem risco por eventos extremos.

$$
Q_{n}=\frac{Q_{i}-\bar{x}}{\sigma}
$$

em que:

$Q_{n}$ é a vazão normalizada,

$Q_{i}$ é a vazão observada em um determinado ano,

$\bar{x}$ é a média aritmética e $\sigma$ é o desvio padrão.

Os índices de ameaça de cheia foram encontrados a partir da média mais desvio padrão $(\bar{x}+\sigma)$, e média mais $2 *$ desvio padrão $(\bar{x}+2 * \sigma)$; e os de ameaça de seca foi a partir da média menos desvio padrão $(\bar{x}-\sigma)$, e média menos $2 *$ desvio padrão $(\bar{x}-2 * \sigma)$. A partir dos cálculos, os índices de ameaça foram classificados, conforme os itens a seguir:

a) Sem Risco de Ameaça: Classificação “0” (zero);

b) Ameaça Muito Baixa: Classificação de $\pm 0,1$ à $\pm 0,125$;

c) Ameaça Baixa: Classificação de $\pm 0,126$ à $\pm 0,25$;

d) Ameaça Moderada: Classificação de $\pm 0,26$ à $\pm 0,5$;

e) Ameaça Alta: Classificação de $\pm 0,51$ à $\pm 0,75$; e

f) Ameaça Muito Alta: Classificação de $\pm 0,76$ à \pm 1 (um).

Obs.:

(-) refere-se à classificação para os eventos de seca; e

(+) para eventos de cheia

Para todos os valores de vazão normalizada entre $(\bar{x})$ e $(\bar{x}-\sigma)$, o evento é considerado de pouca seca; se os valores estiverem exatamente na faixa de $(\bar{x}-\sigma)$, o evento considerado é de seca moderada; se os valores estiverem entre $(\bar{x}-\sigma)$ e $(\bar{x}-2 * \sigma)$, o evento considerado é de seca extrema; e se os valores forem maiores ou iguais a $(\bar{x}-2 * \sigma)$, o evento é de seca altamente extrema. Fazendo as mesmas relações para o período de cheia, para os valores de vazão entre $(\bar{x})$ e $(\bar{x}+\sigma)$, o evento é considerado de pouca cheia; se os valores estiverem exatamente na faixa de $(\bar{x}+\sigma)$, o evento considerado é de cheia moderada; se os valores estiverem entre $(\bar{x}+\sigma)$ e $(\bar{x}+2 * \sigma)$; o evento considerado é de cheia extrema; e por fim, se os valores forem maiores ou iguais a $(\bar{x}+2 * \sigma)$, o evento é de cheia altamente extrema.

\subsection{Ameaça ambiental}

Essa etapa foi feita em função das anteriores, para a elaboração de uma matriz de risco a partir dos aspectos socioeconômicos e ambientais para cada município. A partir do IVS e da Ameaça ambiental encontrou-se o Risco socioeconômico e ambiental, conforme Equação 3.

$$
\text { Risco }=I V S \times \text { Ameaça }
$$


Após a determinação do Risco socioeconômico e ambiental, utilizou-se o Índice do Quadro 1 para indicar a variação do Índice de Risco de cada município, ou seja, Sem Risco (0), Risco Muito Baixo ( [0,125 à 0,25[ ), Risco Baixo ( [0,25 à 0,50[ ), Risco Moderado ([0,5 à

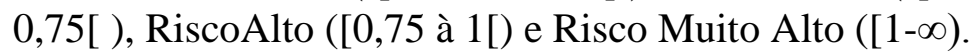

Quadro 1. Índice de risco socioeconômico ambiental.

\begin{tabular}{|c|c|c|c|c|}
\hline \multicolumn{5}{|c|}{ Vulnerabilidade (IVS) } \\
\hline \multirow{6}{*}{ 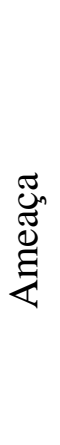 } & & 0 & 0,5 & 1 \\
\hline & 0 & 0 & 0 & 0 \\
\hline & 0,25 & 0 & 0,125 & 0,25 \\
\hline & 0,50 & 0 & 0,25 & 0,5 \\
\hline & 0,75 & 0 & 0,375 & 0,75 \\
\hline & 1 & 0 & 0,5 & 1 \\
\hline
\end{tabular}

\begin{tabular}{|ll|}
\hline \multicolumn{1}{|c|}{ Legenda de Risco: } \\
\hline \multicolumn{1}{|}{} & Sem Risco \\
\hline & Risco Muito Baixo \\
\hline & Risco Baixo \\
\hline & Risco Moderado \\
\hline & Risco Alto \\
\hline & Risco Muito Alto \\
\hline
\end{tabular}

\section{RESULTADOS E DISCUSSÃO}

Na Figura 1, os indicadores que compõe o Índice de Vulnerabilidade Social (IVS) tiveram comportamento diferenciado nos estados, pois o diagnóstico foi semelhante somente entre o Amazonas e Pará, que são os mais densamente povoados, com IVS moderado $(0,5)$ somente no indicador PI. Nos estados com menor densidade demográfica, Roraima, Acre e parte do Mato Grosso o IVS foi alto (1).

$\mathrm{Na}$ Figura 1, o indicador escolaridade (PE) apresentou vulnerabilidade entre 0 (zero), intensidade baixa, em Rondônia e Roraima, e 0,5, intensidade moderada, no Mato Grosso e Acre. Tal indicador é importante para diminuição da vulnerabilidade dos municípios, caminho ainda a ser alcançado pela maioria dos municípios da Amazônia. Entretanto, nas capitais a escolaridade não é o ponto mais crítico, porém, é um indicador decisivo, mostrado também nos estudos de Sena et al. (2012). Com o planejamento territorial, somente alguns dos indicadores de vulnerabilidade podem ser modificados, como o PE, PR e PU, que quando possuem uma classificação elevada aumentam a vulnerabilidade, conforme a Tabela 1.

$\mathrm{O}$ indicador rendimento (PR), como indicador de vulnerabilidade, também foi identificado em vários estudos, como o de Alves (2006) que encontrou alta vulnerabilidade social em áreas pobres com degradação ambiental, e o de Gonçalves et al. (2014) que também afirmaram ser o grupo com menor rendimento o que apresenta maior vulnerabilidade. Associado à vulnerabilidade socioeconômica, Pinho et al. (2015) estudaram a relação com os eventos meteorológicos, e concluíram que eles exacerbam os riscos, aumentando a vulnerabilidade do sistema, onde a pobreza é o índice que causa a mais alta vulnerabilidade, concordando com os dados encontrados na Figura 1.

O indicador de urbanização apresentou índice alto $(1,0)$ em todos os estados, ou seja, em média $90 \%$ da população vive em áreas urbanas e quando essas áreas estão suscetíveis a eventos extremos, a população fica mais vulnerável, e com isso, correm mais riscos. Tanto Silva Dias et al. (2005) como Marengo et al. (2008) afirmam que a vulnerabilidade da população urbana é maior com valores fluviométricos mais elevados, ou seja, eventos extremos de cheia. Contudo, Sena et al. (2012), detectou que áreas urbanizadas com possibilidade de cheias possuem alta vulnerabilidade, ficando em uma situação ainda mais grave se a população tiver baixo rendimento. 
O indicador PC pode ser modificado em menor escala através do controle de natalidade e, com isso, melhorando o índice de vulnerabilidade, porém o PF e o PI são indicadores que não podem ser modificados por nenhum tipo de programa ou políticas públicas. Assim, sempre serão indicadores preponderantes no aumento da vulnerabilidade da população.

O PI apresentou índices de baixo à moderado, conforme a Figura 1. Entretanto, as políticas para esse tipo de índice não buscam a diminuição da vulnerabilidade, pois não existe como fazêlo, mas sim, melhorar a forma de adaptação que esse grupo terá para que a ameaça ambiental diminua o risco socioeconômico e ambiental.
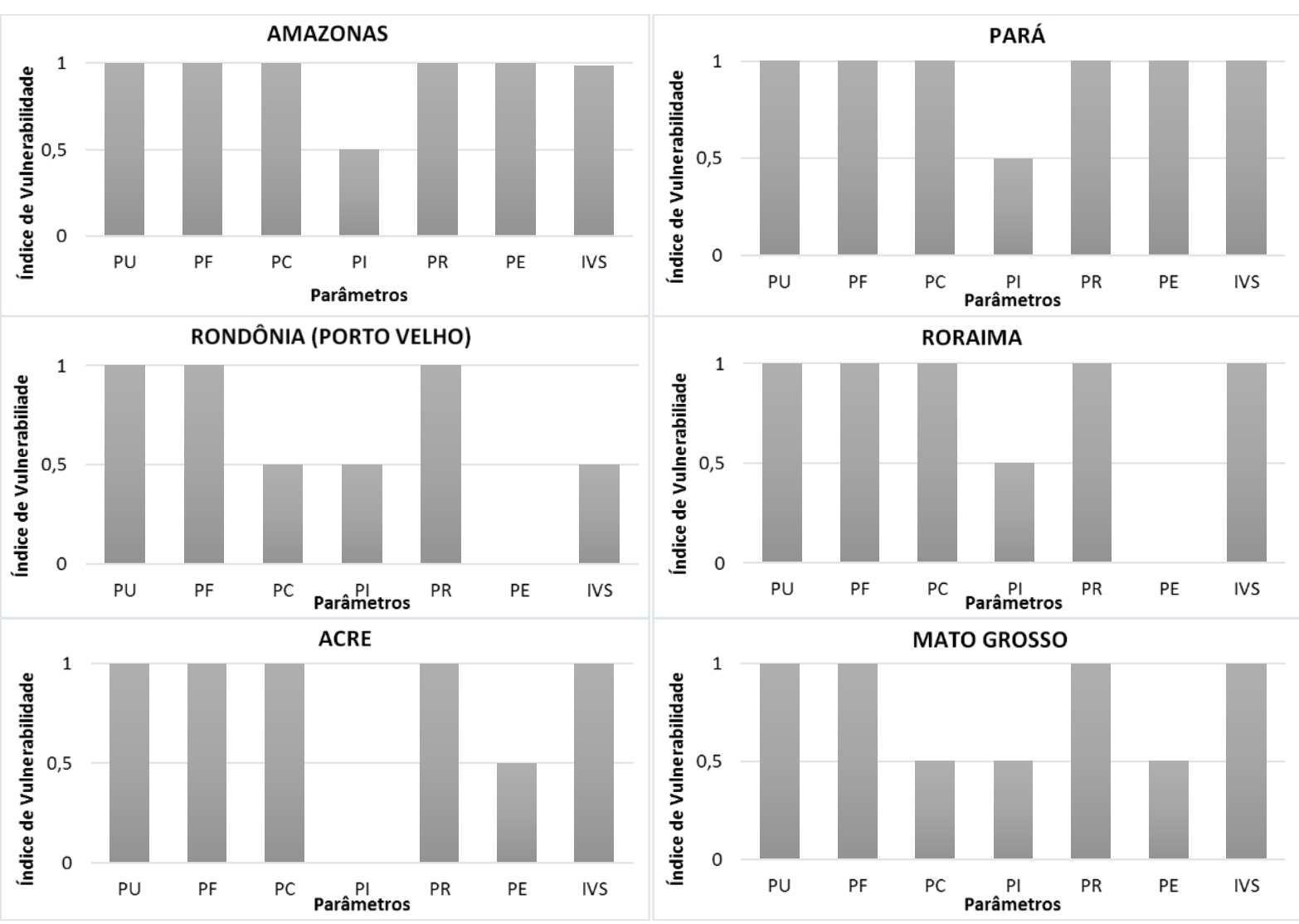

Figura 1. Índice de Vulnerabilidade Socioeconômico nos estados da bacia Amazônica.

A Figura 2 representa o risco socioeconômico e ambiental para cada estado nos anos de estiagem e de enchente, e conforme os estudos de Gonçalves et al. (2014), Sena et al. (2012), Marengo et al. (2008) e Silva Dias et al. (2005), esses riscos são diferentes a cada evento extremo, devido não somente ao tipo do evento, mas também a sua frequência, intensidade, tempo e espaço de ocorrência.

Somente nos estados de Rondônia e Roraima, a seca prevaleceu nos municípios estudados, com $58 \%$ e $52 \%$, respectivamente. Além disso, pode-se notar que esses estados são os que estão mais em risco do que os demais, seja pelas cheias ou secas, pois somente em $6 \%$ dos municípios, a população fica fora de risco. Já o estado do Pará é mais afetado pelas cheias, com $62 \%$ dos municípios, seguido pelo estado do Acre com 56\%, Amazonas com 54\% e Mato Grosso com 49\%, comprovado, também em estudos como Sena et al. (2012), Marengo et al. (2008) e Silva Dias et al. (2005). Os estados do Acre e Mato Grosso são os que apresentaram menor risco à população com $19 \%$ e $17 \%$ dos municípios, respectivamente.

O risco socioeconômico e ambiental no Estado de Rondônia foi avaliado somente através do município de Porto Velho e pode-se notar, conforme a Figura 2, que a frequência de risco foi a menor quando comparada com os outros estados, pois apresentou 58\% de risco muito 
baixo de seca e $38 \%$ de risco muito baixo de cheia, e quando associado ao IVS moderado $(0,5)$ (Figura 1), mostra que os eventos extremos afetam menos a população.

Somente no Estado do Amazonas o risco de cheia foi alto, porém quando se trata do risco alto de seca, mais estados são afetados, como em Roraima e Mato Grosso. Contudo, os estados com maior densidade demográfica são os que possuem maior risco socioeconômico e ambiental.

Se os riscos alto e moderado de cheia forem somados em todos os estados, a população com maior risco encontra-se no Amazonas, com 11\% dos municípios estudados. Isso deve-se ao fato que o Amazonas possui um excesso de água em seu balanço hídrico, então com aumento das chuvas as cheias são mais frequentes, indo ao encontro dos estudos de Sena et al. (2012) que também afirmam que as zonas mais vulneráveis estão relacionadas principalmente com as inundações. O Estado do Pará apresenta $7 \%$ dos municípios com risco de cheia moderada, que também apresenta um excesso de água em seu balanço hídrico.

Quando se analisa a época de seca, percebe-se que no Mato Grosso ocorreu a maior intensidade de risco moderado de seca e risco alto de seca, com 5\% dos municípios. Isso se deve muito ao tipo de clima da região que é árido, consequentemente ocorrendo um maior déficit de água no balanço hídrico na região.

Analisando, separadamente cada município da Figura 3, percebe-se que nenhum dos 47 municípios dos 6 estados apresentaram risco socioeconômico e ambiental maior que o índice moderado $(0,5)$, tanto em eventos de seca como em eventos de cheia. No Amazonas, conforme mostra a Figura 3, nos municípios de Santo Antônio do Içá, Manicoré e Manacapuru, em média, a população não apresenta riscos relacionados aos eventos extremos de seca, entretanto, apresentam risco moderado $(0,5)$ de cheia. E nos municípios de Jutaí e Envira, a população não sofreu risco com relação aos eventos de cheia. Como já mencionado anteriormente, o estado do Amazonas é o mais influenciado pelas cheias. Entretanto, dos 25 municípios estudados no Amazonas, $64 \%$ foram mais afetados pelos riscos socioeconômicos e ambientais devido às secas. Isso prova que existem variações muito grandes ao longo do Rio Amazonas, motivo pelo qual torna-se foco de estudos de vários cientistas, como mostrado por Gonçalves et al. (2014), Filizola et al. (2014), Sena et al. (2012) e Alves (2006), entre outros.

Na sub-bacia do Rio Negro, todos os municípios estudados apresentaram ameaça de cheia em 2009, quando ocorreu uma das maiores enchentes das últimas décadas. No Estado do Pará e Amazonas esse cenário foi o mesmo, pois, todos os municípios também apresentaram ameaça de cheias, indo ao encontro dos estudos de Marengo et al. (2012) onde as enchentes de 2009 foram muito intensas no norte e leste da Bacia Amazônica. Já em 2012, outro ano de cheia intensa, os municípios da Amazônia ocidental não apresentaram resultados tão afirmativos, porém os municípios do Noroeste, que ficam no rio Negro, mostraram essa intensa cheia, corroborando com os estudos de Satyamurty et al. (2013) e Marengo et al. (2013).

$\mathrm{Na}$ cidade de Manaus, $99 \%$ da população vive em áreas urbanizadas e 35\% na pobreza, conforme o IBGE (2010), porém a escolaridade é elevada com o IVS moderado. A ameaça ambiental possui um índice baixo na cidade de Manaus, ou seja, os eventos extremos não a afetam substancialmente, fazendo com que o risco socioeconômico e ambiental não seja tão elevado. Essa afirmação vai ao encontro dos estudos de Pinho et al. (2015) pois eles afirmam que são as regiões mais remotas e não as capitais, por apresentarem infraestrutura básica eficaz, que apresentam maior vulnerabilidade e com isso correm maiores riscos socioeconômicos e ambientais, ocasionando melhor qualidade de vida, que é o objetivo dos planos diretores dos municípios. 
Riscos socioeconômico e ambientais em municípios ...

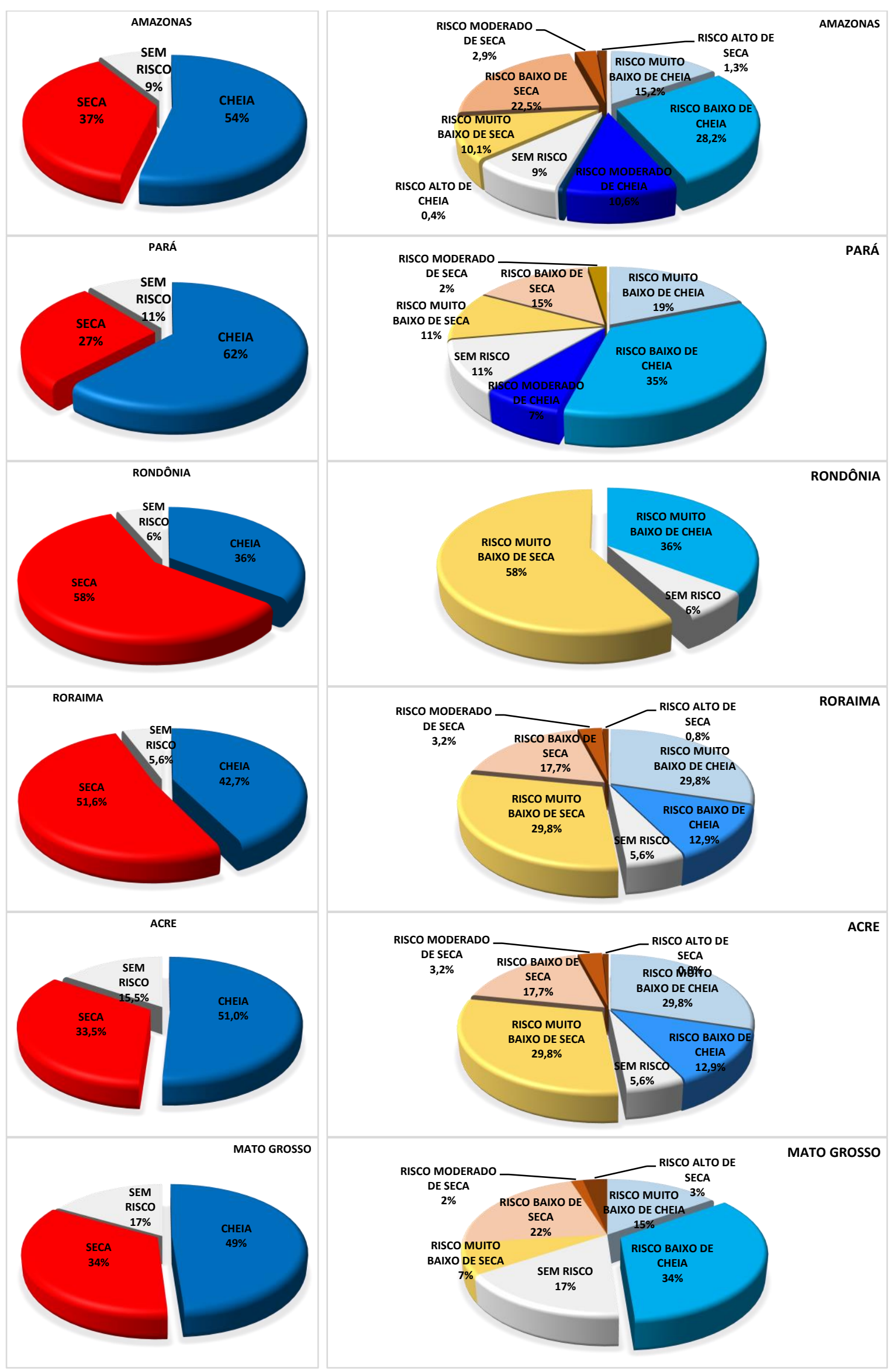

Figura 2. Risco socioeconômico e ambiental durante a estiagem e enchente. 


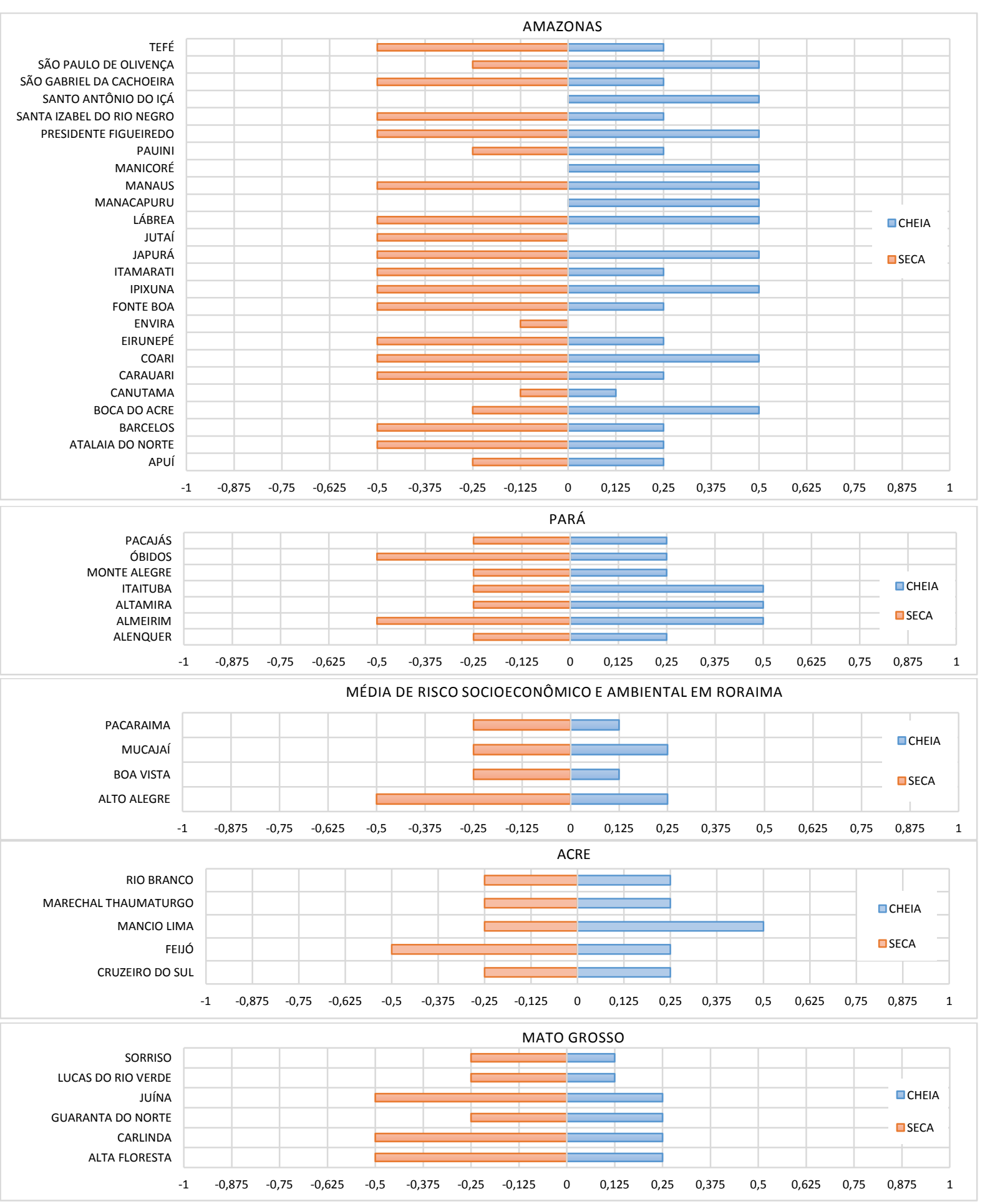

Figura 3. Média do Risco socioeconômico e ambiental nos municípios da Bacia Amazônica.

Analisando as capitais dos outros estados, como Porto Velho (RO) (Figura 2), Boa Vista (RR) (Figura 3c) e Rio Branco (AC) (Figura 3d), o risco socioeconômico e ambiental foi pequeno, variando entre muito baixo e baixo tanto durante os períodos de seca como nos periodos de cheia, sendo contrário aos estudos de Sena et al. (2012), já que estes autores afirmam que as cheias causam mais riscos às populações.

Fazendo as análises dos riscos socioeconômico e ambiental para cada município, percebeu-se que no Pará (Figura 3b), 43\% dos municípios apresentaram risco moderado durante 
as cheias e somente $28 \%$ durante as secas, e $72 \%$ obteve risco baixo na época seca e $57 \%$ de risco baixo na época cheia, perfil diferenciado de todos os outros estados, que apresentaram maior risco na época seca, resultado que vai de encontro ao de Sena et al. (2012).

Nos municípios do Estado do Pará, os Índices do IVS apresentaram valores de moderado $(0,5)$ a alto $(1,0), 100 \%$ desses municípios apresentaram índices altos de PU, PF e PR, conforme mostrado na Figura 1. As ameaças ambientais apresentaram índice moderado, e quando relacionado com o IVS alto, minimizou os riscos socioeconômicos e ambientais apresentados na Figura 3b.

Conforme a Figura 3, os municípios de Roraima foram os que apresentaram os menores riscos sócioeconômicos e ambientais, principalmente na época de cheia, e analisando a Figura 1, pode-se afirmar que a vulnerabilidade é o que mais afeta tais municípios e não a ameaça, pois o IVS foi alto (1).

No Acre, conforme a Figura 3d, os riscos socioeconômicos e ambientais apresentaram o mesmo perfil entre os eventos extremos de cheia e seca, pois $20 \%$ dos municípios obtiveram índices moderados e $80 \%$ de risco baixo, e relacionando com o IVS, na Figura 1, pode-se afirmar que a vulnerabilidade é a que mais afeta os riscos, sendo diferente dos municípios do Mato Grosso, já que os riscos sócioeconômicos e ambientais são mais afetados pelas ameaças de seca e cheia do que pela vulnerabilidade, indo de encontro aos estudos de Sena et al. (2012).

\section{CONCLUSÕES}

Vale destacar que se deve ter cuidado na utilização de indicadores nesse tipo de pesquisa, entretanto os dados do censo demográfico de 2010 do IBGE possui um escopo que proporcionou três dimensões ao IVS: infraestrutura urbana, capital humano e renda e trabalho, pontos essenciais para determinar a vulnerabilidade.

Todas as variações das componentes do balanço hídrico, como a vazão, precipitação e evaporação ocorrem no tempo e no espaço, e quando associadas com eventos extremos pluviométricos, ocasionam ameaças às populações, principalmente as com maior vulnerabilidade, causando assim riscos socioeconômicos e ambientais. A área mal urbanizada na Amazônia, quando associada com outros indicadores, principalmente ambientais, como eventos extremos de precipitação, pode causar danos irreversíveis à população, sendo assim, pode-se comprovar nos gráficos que a vulnerabilidade por área de urbanização foi alta, com índice chagando a 3. Entretanto, outros fatores também são responsáveis pela vulnerabilidade, como a pobreza, que em todos os estados foi elevada, com índice 3.

Assim, concluiu-se que nos municípios que são banhados pelo Amazonas a população possui um risco socioeconômico e ambiental de moderado a muito forte, devido a todos os estados possuírem um índice alto de vulnerabilidade quanto à acelerada e desordenada urbanização e à pobreza (1) e ameaça quanto às enchentes e estiagens também variando de moderada a alta $(0,5$ e 1$)$. Ainda se pode afirmar que as cheias são as que provocam maiores riscos às populações, que os estados com maior vulnerabilidade são os com maior área espacial, Amazonas e Pará, e os estados com maior ameaça foram os menores espacialmente, Roraima e Acre.

A relação dos riscos de enchente e seca depende das características climáticas e tipo de vegetação em cada região. Rondônia e Roraima são os estados que mais sofrem com as queimadas, assim os maiores riscos são os da seca. Entretanto, entre os estados com maior dimensão, os riscos maiores são de enchentes. Por fim o estado do Mato Grosso, na Figura 3, apresenta mais municípios com risco de seca, pois encontra-se na parte central do Brasil, onde o clima é mais seco.

Assim, para que o risco socioeconômico e ambiental seja diminuído na região estudada, as políticas públicas devem ser aplicadas em todas as regiões, independentemente da localidade, 
sejam em função da urbanização, rendimento, escolaridade ou mesmo acontecimento de eventos extremos, pois a associação entre eles, provocam riscos elevados.

\section{AGRADECIMENTOS}

Agradecemos a Agência Nacional de Águas (ANA) e ao Instituto Brasileiro de Geografia e Estatística (IBGE) pela disponibilização dos dados utilizados nesse trabalho, além do Programa de Pós-graduação de Ciências Ambientais da Universidade Federal do Pará, Empresa Brasileira e Agropecuária (EMBRAPA) e Museo Emílio Goeldi (MPEG), pela oportunidade na conclusão do doutorado.

\section{REFERÊNCIAS}

ACSELRAD, H.; HERCULANO, S.; PÁDUA, J. A. A justiça ambiental e a dinâmica das lutas socioambientais no Brasil - uma introdução. In: ACSELRAD, H.; HERCULANO, S.; PÁDUA, J. A. (Orgs.). Justiça ambiental e cidadania. Rio de Janeiro: Relume-Dumará; 2004. p. 14.

ALVES, H. P. F. Vulnerabilidade socioambiental na metrópole paulistana: uma análise sóciodemográfica das situações de sobreposição espacial de problemas e riscos sociais e ambientais. Revista Brasileira de Estudos da População, v. 23, n. 1, p. 43-59, 2006. http://dx.doi.org/10.1590/S0102-30982006000100004

AGÊNCIA NACIONAL DE ÁGUAS - ANA (Brasil). Hidroweb. Disponível em: http://hidroweb.ana.gov.br/default.asp. Acesso em: 02 jul. 2013.

ARAUJO, R.; LÉNA, P. Da predação a sustentabilidade na Amazônia: a difícil metamorfose. In: ARAÚJO, R.; LÉNA, P. (Eds.). Desenvolvimento sustentável e sociedades na Amazônia. Belém: MPEG, CNPQ, PPG-7, 2010.

ASSAD, L. Um planeta que parece cada vez mais inquieto. Ciência e Cultura, v. 63, n. 1, p. 9-11, 2011. http://dx.doi.org/10.21800/S0009-67252011000100004

BRAGA, T. M.; OLIVEIRA, E. S. de; GIVISIEZ, G. H. N. Avaliação de metodologias de mensuração de risco e vulnerabilidade social a desastres naturais associados à mudança climática. São Paulo em Perspectiva, v. 20, n. 1, p. 81-95, 2006.

BRAUCH, H. G. Threats, challenges, vulnerabilities and risks in environmental and human security. Bonn: UNU- EHS, 2005. 104 p.

CARTIER, R.; BARCELLOS, C.; HÜBNER, C.; PORTO, M. F. Vulnerabilidade social e risco ambiental: uma abordagem metodológica para avaliação de injustiça ambiental. Cadernos de Saúde Pública, v. 25, n. 12, p. 2695-2704, 2009. http://dx.doi.org/10.1590/S0102-311X2009001200016

ESPINOZA, J. C.; RONCHAIL, J.; GUYOT, J. L.; JUNQUAS, C.; VAUCHEL, P.; LAVADO, W. et al. Climate variability and extreme drought in the upper Solimões River (western Amazon Basin): Understanding the exceptional 2010 drought. Geophysical Research Letters, v. 38, p. 1-6, 2011. http://dx.doi.org/10.1029/2011GL047862

ESTEVES, C. J. O. Risco e vulnerabilidade socioambiental: aspectos conceituais. Caderno IPARDES. v. 1, n. 2, p. 62-79, 2011. 
FILIZOLA, N.; LATRUBESSE, E. M.; FRAIZY, P.; SOUZA, R.; GUIMARÃES, V.; GUYOT, J.-L. Was the 2009 flood the most hazardous or the largest ever recorded in the Amazon? Geomorphology, v. 2015, p. 99-105, 2014. https://doi.org/10.1016/j.geomorph.2013.05.028

FLECK, A. C.; WAGNER, A. A mulher como principal provedora do sustento econômico familiar. Psicologia em Estudo, 8, n. spe, p. 31-38, 755-783. 2003. http://dx.doi.org/10.1590/S1413-73722003000300005

GONÇALVES, K. S.; SIQUEIRA, A. S. P.; CASTRO, H. A. de; HACON, S. S. Indicador de vulnerabilidade sociambiental da Amazônia Ambiental. O caso do município de Porto Velho, Rondônia, Brasil. Ciência \& Saúde Coletiva, v. 19, n. 9, p. 3809-3817, 2014. http://dx.doi.org/10.1590/1413-81232014199.14272013

GUIMBERTEAU, M.; RONCHAIL, J.; ESPINOZA, J. C.; LENGAIGNE, M.; SULTAN, B.; POLCHER, J. et al. Future changes in precipitation and impacts on extreme streamflow over Amazonian sub-basins. Environmental Research Letters, v. 8, n. 1, p. 1748-9326, 2013.

INSTITUTO BRASILEIRO DE GEOGRAFIA E ESTATÍSTICA - IBGE. Censo 2010. 2010. Disponível em: http://www.ibge.gov.br/home/. Acesso em: 02 ago 2013.

KATAYAMA, T. Lifelines in urban areas: To what extent can their importance during disasters be justified? In: DISASTER MANAGEMENT IN METROPOLITAN AREAS FOR THE $21^{\text {st }}$ CENTURY. 1993, Nagoya. Proceedings... Nagoya: UN, 1993. p. 295-303.

LEAL, S. V.; SOUZA, E. B. Desastres naturais sobre a Amazônia e Nordeste Brasileiro associados às enchentes e inundações: o caso de 2009. In: ENCONTRO SULBRASILEIRO DE METEOROLOGIA, 4., 2011, Pelotas-RS. Anais... Pelotas: SBMET, 2011.

MARENGO, J. A.; LINCOLN, M.; ALVES, W.; SOARES, R.; RODRIGUEZ, D. A.; CAMARGO, H. et al. Two Contrasting Severe Seasonal Extremes in Tropical South America in 2012: Flood in Amazonia and Drought in Northeast Brazil. Journal of Climatology, v. 26, 2013. https://doi.org/10.1175/JCLI-D-12-00642.1

MARENGO, J. A.; TOMASELlA, J.; SOARES, W. R.; ALVES, L. M.; NOBRE, C. A. Extreme climatic events in the Amazon basin. Theoretical and Applied Climatology, v. 107, p. 73-85, 2012. http://dx.doi.org/10.1007/s00704-011-0465-1

MARENGO, J. A.; NOBRE, C. A.; TOMASELLA, J.; OYAMA, M. D.; OLIVEIRA, G.V.S.; OLIVEIRA, R. et al. The Drought of Amazonia in 2005. Journal of Climatology, v. 21, n. 1, 2008. https://doi.org/10.1175/2007JCLI1600.1

O'RIORDAN, T.; STOLL-KLEEMANN, S. Biodiversity, sustainability, and human communities: protecting beyond the protected. Cambridge; New York: Cambridge University Press, 2002.

PARRY, L.; PERES, C. A.; DAY, B.; AMARAL, S. Rural-urban migration brings conservation threats and opportunities to Amazonian water sheds. Conservation Letters, v. 3, p. 251-259, 2010. http://dx.doi.org/10.1111/j.1755-263X.2010.00106.x

PINHO, P. F.; MARENGO, J. A.; SMITH, M. S. Complex socio-ecological dynamics driven by extreme events in the Amazon. Regional Environmental Change, v. 15, p. 643-655, 2015. http://dx.doi.org/10.1007/s10113-014-0659-z 
ROLIM, P. A. M.; SANTOS, D. M.; ROCHA, E. J. P. Variabilidade da precipitação na Amazônia: implicações socioeconômicas. In: CONGRESSO BRASILEIRO DE METEOROLOGIA, 14., 2006, Florianópolis. Anais... Rio de Janeiro: SBMET, 2006.

QUARANTELLI, E. L.; POPOV, K. Proceedings of the United States-Former Soviet Union Seminar on Social Science Research on Mitigation for and Recovery from Disaster and Large Scale Hazards, Volume I: The American Participation. Newark: Disaster Research Center; University of Delaware, 1994.

SATYAMURTY, P.; COSTA, C. P. W.; MANZI, A. O.; CANDIDO, L. A. A quick look at the 2012 record flood in the Amazon Basin. Geophysical Research Letters, v. 40, p. 13961401, 2013. http://dx.doi.org/10.1002/grl.50245

SENA, J. A.; FREITAS, M. A. V.; BERRÊDO, D.; FERNANDES, L. C. Evaluation of Vulnerability to Extreme Climatic Events in the Brazilian Amazonia: Methodological Proposal to the Rio Acre Basin. Water Resources Management, v. 26, p. 4553-4568, 2012. http://dx.doi.org/10.1007/s11269-012-0166-2

SILVA DIAS, M. A. F.; COHEN, J. C. P.; GANDU, A. W. Interações entre nuvens, chuvas e a biosfera na Amazônia. Acta Amazônica, v. 35, n. 2, p. 215-222, 2005. http://dx.doi.org/10.1590/S0044-59672005000200011

SZLAFSZTEIN, C.; MARQUES, O.; MAIA, H.; PRETTE, M.; FISCHENICH, P.; ALTIERI, F. Referências metodológicas para mapeamento de riscos naturais na Amazônia: mapeando as vulnerabilidades. Brasília: MMA/GTZ, 2010. 60 p. 